\title{
Aberrant Expression of the Multi-CSF Gene in Hematopoietic Precursor and Stem Cell Lines Initiates Leukemogenic Progression
}

\author{
C. Laker ${ }^{1}$, C. Stocking ${ }^{1}$, N. Kluge ${ }^{1}$, M.J. Franz ${ }^{1}$, G. Beck-Engeser ${ }^{1}$, E. Spooncer ${ }^{2}$,
} M. Dexter ${ }^{2}$, and W. Ostertag ${ }^{1}$

\section{A. Introduction}

The most common feature of many malignancies is the escape of transformed cells from normal growth requirements. In hemopoiesis, a series of well-characterized glycoproteins (colony-stimulating factors, CSFs) tightly control the survival, proliferation, differentiation, and functional activation of all blood cells [1]. In addition, the differentiation hierarchy of pluripotent stem cells, capable of selfrenewal and differentiation into all lineages of myelopoiesis and lymphopoiesis, to terminally differentiated cells via multipotent precursor cells may be traced by surface markers and functional assays. Thus, the ability to determine the differentiation stage of a cell and an understanding of the molecules that trigger cell division make haemopoiesis a suitable system to study the initiation of malignant progression, i. e., leukemogenesis.

In the data presented here, we will focus on the following aspects:

1. Can aberrant expression of a CSF gene in a factor-dependent cell confer factor independence?

2. Is the acquisition of factor independence coincident with tumorigenesis?

3. If not, what are the other parameters that determine tumorigenic potential of such cells?

4. Can differences between stem and progenitor cells in tumorigenic progression

1 Heinrich-Pette-Institut für Experimentelle Virologie und Immunologie an der Universität Hamburg, Martinistrasse 52, 2000 Hamburg 20, FRG

${ }^{2}$ The Paterson Institute for Cancer Research, Manchester M20 9BX, UK following constitutive expression of CSF genes be detected?

To address these questions, we chose two different types of hemopoietic cell lines as model systems. The FDC-Pmix stem cell lines [2] retain a normal or nearly normal karyotype and their differentiation potential is close to that of normal myeloid stem cells. Indeed, some FDCPmix cells can repopulate the hematopoietic system of irradiated animals. The second cell types, FDC-P1 and FDC-P2, have a more restricted differentiation pattern, an abnormal karyotype, and most likely are representatives of myeloid progenitor cells [3].

\section{B. Retroviral Transfer Into Hematopoietic Target Cells}

The FDC-P progenitor cell lines were derived from normal long-term bone marrow cultures (Dexter-type cultures), whereas the FDC-Pmix stem cell lines were established from bone marrow cultures infected with Moloney murine leukemia virus (Mo-MuLV) and a recombinant retroviral vector carrying the avian v-src oncogene. The stromal cells of the long-term cultures express the src oncogene product pp60 v-src, whereas the FDC-Pmix cells do not. They do, however, express the ecotropic MoMuLV used as helper virus to provide viral spread of the replication defective src virus in the original cultures. In order to overcome retroviral interference by Mo-MuLV-infected FDC-Pmix cells, we used the amphotropic PA317 packaging cell line to assemble vector-carrying pseudotypes suitable for infection of the 
target cells. Since the FDC-P1 progenitor cell line does not express ecotropic retroviral sequences, the ecotropic $\psi 2$ packaging cell line was used as a viral source.

The vectors used for transfer of either the granulocyte/macrophage (GM)-CSF or multi-CSF gene are based on the myeloproliferative sarcoma virus (MPSV), which has a broader host range as compared with Mo-MuLV due to mutations in the U3 region of the LTR [4]. All retroviral constructs contain the Tn5 drug resistance gene (Neo) and therefore allow selection with geneticin (G418). The CSF genes either replace the mos oncogene sequences of the native virus or are inserted into the viral genome coding for the gag leader just $3^{\prime}$ of the packaging site [5] (Fig. 1).
To test the transfer efficiency of MPSV-based vectors into hematopoietic cell lines, FDC-P1/2 progenitor cells or FDC-Pmix cells were infected with a limiting amount of virus particles, plated in the presence of exogenous growth factor and selected with G418 $(1.5 \mathrm{mg} / \mathrm{ml})$. The number of G418-resistant colonies was scored 9-16 days after infection and compared with the number of G418-resistant colonies of standard fibroblasts (NIH/3T3) obtained from a simultaneously performed infection by limiting dilution.

Table 1 shows the relative G418 transfer efficiency to hematopoietic target cells as compared with fibroblasts. The data demonstrate that the transfer efficiency into progenitor cells (FDC-P1/2)
Fig. 1. Retroviral vectors. All vectors were based on the myeloproliferative sarcoma virus (MPSV) and have been described previously $[4,5]$

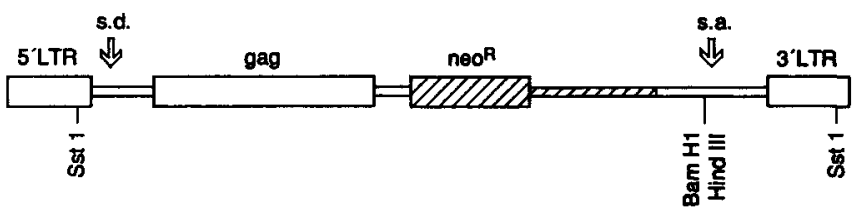

M3 GMV / MuV

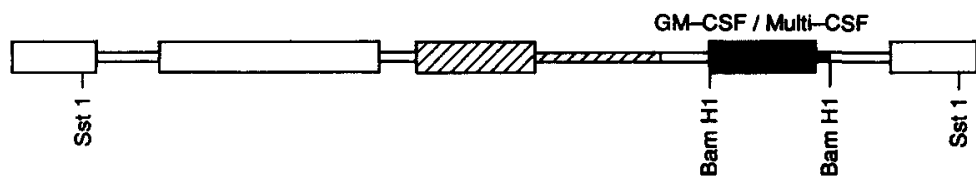

M5 GMV/MuV

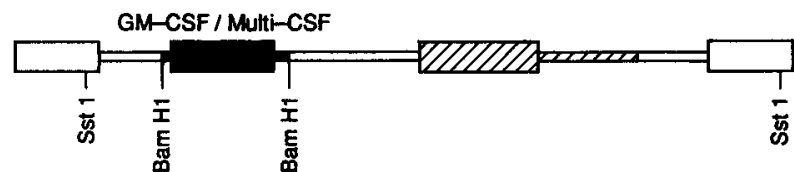

Table 1. Transfer efficiency of Neo into hemopoietic target cells

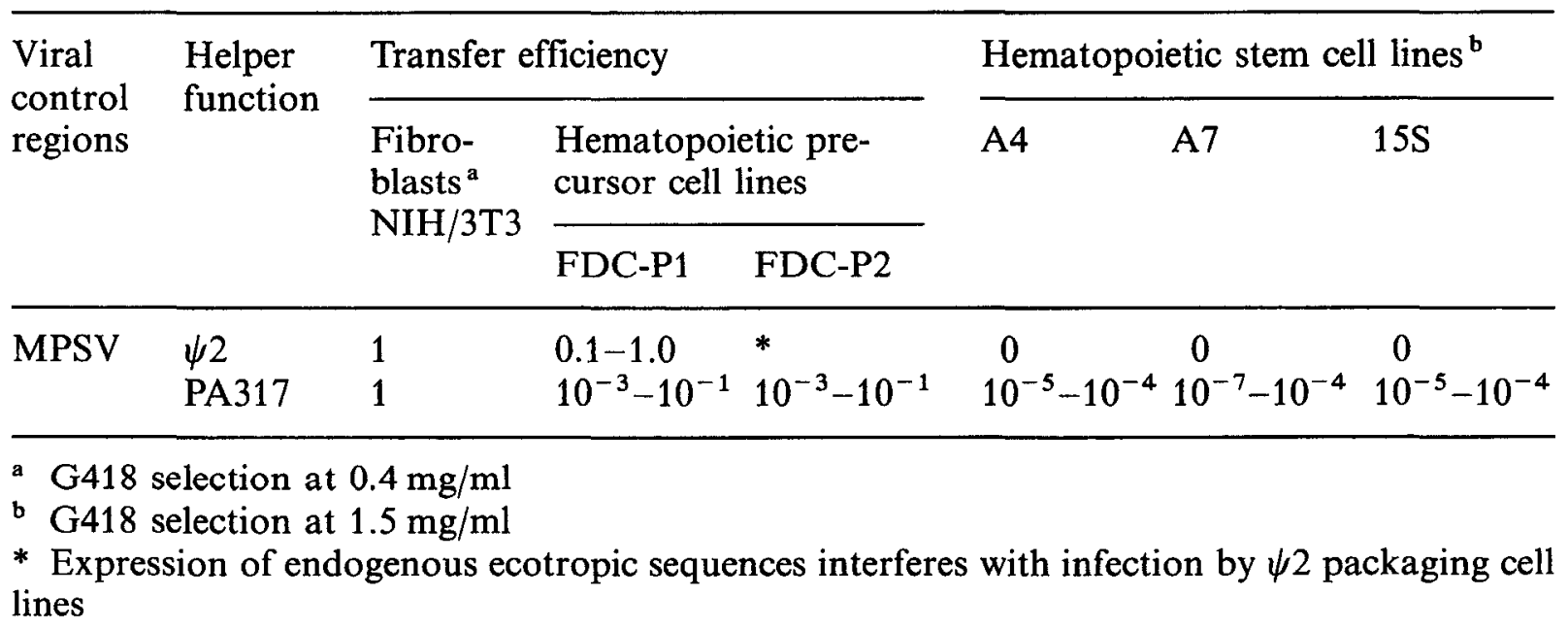


is significantly reduced, as compared with fibroblasts, and decreases dramatically in stem cell lines.

\section{Introduction of a CSF Gene into Factor-Dependent Hemopoietic Target Cells Results in Two Types of Factor Independence}

Infection of growth-factor dependent FDC-P1/2 progenitor cells with either multi-CSF or GM-CSF virus (M3MuV, M3GMV) conferred factor independence to the infected cells. However, the acquisition of growth autonomy after the autogenous production of CSFs appeared to be a consequence of two or more interdependent events. Uninfected cells or cell lines infected with control vectors containing the Neo gene but lacking the growth factor gene showed very low levels of spontaneously occurring growth factor independent mutants: $<10^{-7}$ or $<10^{-9}$ spontaneous mutants could be detected in FDC-P1 or FDC-Pmix cells, respectively. Immediately after introduction of the CSF gene, cells were independent of exogenously supplied CSF at high cell density. They were, however, not truly autonomous, as they still required exogenous CSF at low cell density for cell proliferation (nonautonomous factor independence). These cells often acquired true growth autonomy as a consequence of a second alteration. Growth of such cells was independent of cell density (autonomous). The first, but not the latter type of cells could be inhibited by specific antisera against the CSF.

Analysis of clones picked from primary infections and replated at several time points after infection with MuV demonstrates the shift from nonautonomous factor independence to true autonomy (Fig. 2a, b). Replated 4 weeks after infection in the absence of exogenous growth factor, cells display density-dependent clonability (Fig. 2a). However, after a culture period of several weeks under nonselective conditions (plus WEHI $\mathrm{CM}$ ), replating of the clones gave rise to a more density-independent (linear) cloning pattern when plated in the absence of added growth factor (Fig. 2b). The shift from factor independence, where external stimulation is still required, to true autonomy was accelerated if cloned cultures were kept under selective conditions (minus WEHI CM).

Similar experiments performed by other groups have failed to distinguish the two types of factor independence [6-8], suggesting that infected cells could progress to growth autonomy in one step. Other groups obtained similar results as reported by us [9]. The most striking difference in our work was the use of retroviral vectors designed in our laboratory that express only low levels of the CSF gene product. We therefore designed an alternative construct (M5GMV) which permits a 100 -fold increase in GM-CSF production, as compared with M3GMV. In the primary infection with the M5 construct, the infected cells selected for growth-factor independence (minus WEHI CM) showed a linear cloning pattern. The number of factor-independent clones obtained was, however, considerably lower than that obtained by selection for Neo colonies in the presence of WEHI-conditioned medium (Fig. 3). Analysis of clones picked from various cell densities plated under growth factor selection or G418 selection demonstrated that approximately $50 \%$ of the clones were already autonomous. These data thus suggest that the level of growth factor produced enhances the selective advantage of a second genetic event that leads to autonomous factor independence. If high levels of CSF production are necessary for the shift to growth autonomy, we would predict that autonomous clones should show high or increased release of growth factor. We were, however, unable to detect a significant increase of secreted CSF molecules accompanying the acquisition of growth autonomy.

Our results are, however, consistent with the hypothesis that specific alterations of the export of growth factors or 


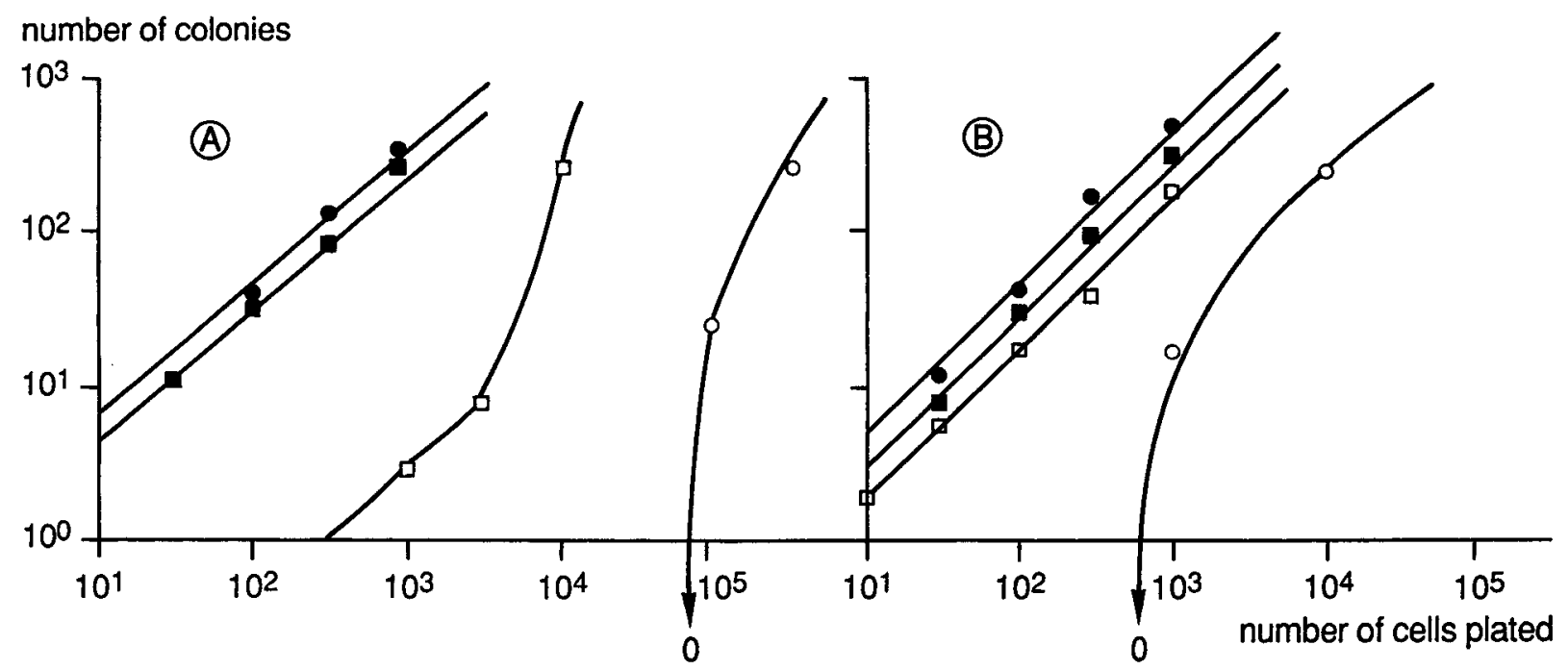

Fig. 2. Clonability of infected FDC-P1 progenitor cells as a function of cell density. The number of clones obtained at different cell densities is plotted on a $\log -\log$ scale; thus, deviation from a slope of +1 reflects non-linear cloning efficiency. Panel A: M3GMV-infected FDC-P1 clone 6 (squares) and clone 4 (circles) recloned 4 weeks after primary infection under plus WEHI CM, plus G418 conditions (closed symbols) or minus WEHI CM selection (open symbols). Panel B: Recloning of the same clones after an additional tissue culture period of 12 weeks (=16 weeks after infection) depicts the shift to autonomous growth

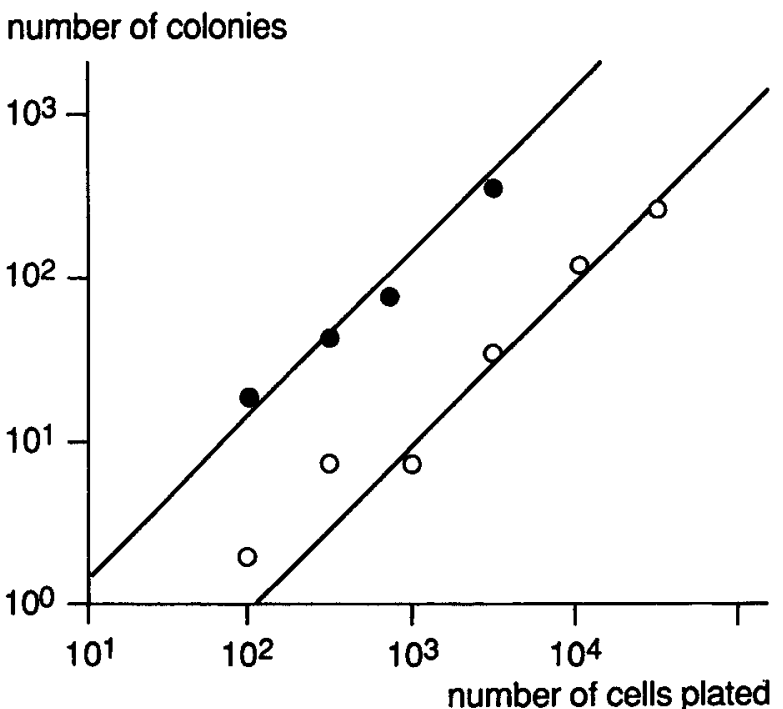

Fig. 3. Primary infection of FDC-P progenitor cells by M5GMV. Cells were infected for $6 \mathrm{~h}$ with supernatant of virus-producing $\psi 2$ fibroblasts, plated in the presence of WEHI $\mathrm{CM}$ and $\mathrm{G} 418(1.5 \mathrm{mg} / \mathrm{ml}$; closed symbols $)$ or absence of added growth factor (open symbols), and scored for colony formation 9 days after infection

the organization of the receptor molecules with respect to internal processing of the growth factor could lead to internal autocrine stimulation and growth autonomy.
To further investigate whether aberrant export of the constitutively expressed CSF gene product contributes to the acquisition of autonomous growth, we designed vectors containing two versions of the multi-CSF gene that differ in the constitution of the leader sequences of the mature protein. The sMuV contains the normal (short) leader sequence resulting in secretion of the mature $\mathrm{CSF}$ molecule, whereas the $1 \mathrm{MuV}$ containing a cDNA clone with a longer leader sequence gives rise to a protein that is predominantly membrane-bound, the body of the protein remaining in the cytosol [10]. Only a small portion $(\sim 10 \%)$ of the molecules is cleaved and secreted.

A significant difference in cloning efficiency under growth factor selection (minus WEHI CM) was observed between FDC-P progenitor cells infected with either short or long leader MuV. FDC-P1 progenitor cells infected with the short leader $\mathrm{MuV}$ showed density-dependent colony formation in the absence of added growth factor when plated immediately after infection (Fig. 4a). In contrast, cells infected with the long leader $\mathrm{MuV}$ showed almost linear (autonomous) 


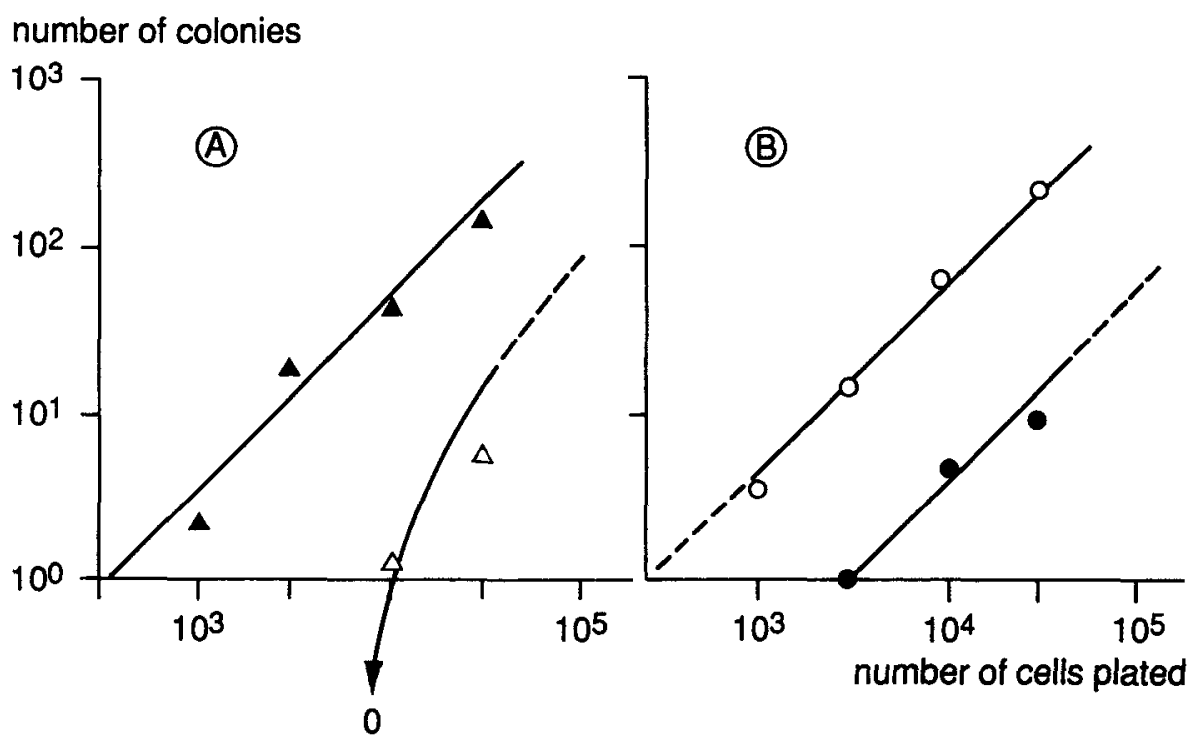

Fig. 4. Primary infection of FDC-P progenitor cells with either M3-sMuV or M3-1MuV. Cells were exposed to virus-containing supernatant for $6 \mathrm{~h}$, washed three times, and plated in semisolid medium at various cell densities either in the presence of WEHI CM and G418 (closed symbols) or absence of WEHI CM (open symbols). Panel $A$ depicts the cloning efficiency of FDC-P1 cells infected with M3-sMuV (short leader sequence) and Panell $B$ the infection of FDC-P2 cells with M3-1MuV (long leader sequence). The proportionally higher clone numbers obtained after selection under minus WEHI conditions, as compared with G418 selection, is most likely due to the 2-day lag in cell death of FDC-P2 cells after removal of WEHI CM

clonability when plated under similar conditions (Fig. 4 b).

Taken together, our data demonstrate that initial factor-independent growth requires receptor-ligand interaction at the cell surface. A second mutation leads to complete autonomous growth of the cell, and both the level and localization of the CSF molecule contribute to the frequency of obtaining such mutations.

\section{The Shift to Growth Autonomy Occurs at a Lower Frequency in Stem Cell Lines}

In contrast to the fast progression of infected FDC-P progenitor cell lines to autonomous factor independence (less than 16 weeks), the FDC-Pmix stem cell lines monitored over a 6-month period displayed a strikingly different behavior. First of all, in primary infections with either $\mathrm{sMuV}$ or $\mathrm{lMuV}$, no clones could be isolated from plates under growth factor selection (cells plated without WEHI $\mathrm{CM}$ ), even when cells were plated at the highest possible cell density $\left(10^{6}\right.$ cells/ $\mathrm{ml}$ ). Follow-up of clones picked from G418 selection (in the presence of WEHI CM) revealed that only one out of 19 clones acquired growth factor autonomy during a 6-month observation period. Interestingly, this particular clone was of $15 \mathrm{~S}$ origin, the parental cell line with the longest culture history and the most committed stem cell feature, having lost its responsiveness to differentiation induction. A4 and A7 stem cells infected with the $\mathrm{M} 3 \mathrm{MuV}$ had to be maintained as bulk cultures under stringent conditions (limiting cell density and lack of added growth factor) to give rise to a few autonomous cell clones after more than 3 months of culture. A subline of the FDCPmix cell clone $15 \mathrm{~S}$ that had been kept in culture continuously for 1 year, however, showed a similar pattern of growth-factor independence and autonomy after infection of $1 \mathrm{MuV}$ as the progenitor lines FDC-P1 or FDC-P2. The 1MuV-infected clones that grow density-dependent show an accelerated shift to growth factor autonomy $\left(10^{-3}\right.$ per cell generation). 
Table 2. Tumorigenicity of M3MuV-infected FDC-P progenitor cell lines

\begin{tabular}{llllll}
\hline $\begin{array}{l}\text { MuV-infected } \\
\text { FDC-P cell clones }\end{array}$ & Clones & $\begin{array}{l}\text { Injected } \\
\text { mice }\end{array}$ & \multicolumn{3}{l}{$\begin{array}{l}\text { No. of tumor-bearing mice } \\
\text { (weeks after inoculation) }\end{array}$} \\
\cline { 5 - 6 }$\left(10^{6}\right.$ cells/mouse) & $(n)$ & $(n)$ & 4 & 8 & 12 \\
\hline $\begin{array}{l}\text { Non-autonomous clones } \\
\text { Autonomous clones }\end{array}$ & 8 & 30 & $0 / 30(0 \%)$ & $3 / 30(10 \%)$ & $19 / 30(63 \%)$ \\
$\begin{array}{l}\text { Controls } \\
\quad \text { Uninfected }\end{array}$ & 2 & 9 & $4 / 9(44 \%)$ & $9 / 9(100 \%)$ & $9 / 9(100 \%)$ \\
$\quad$ Neo V-infected & - & 3 & $0 / 3$ & $0 / 3$ & $0 / 3$ \\
\hline
\end{tabular}

However, infection of this subline with sMuV resulted in clones that shifted at a low rate $\left(10^{-8}\right.$ per cell generation) to growth autonomy. These experiments highlight that both the localization or processing of the CSF molecule and properties of the target cell are important in the acquisition of true autonomy after factor-independent growth by an autocrine mechanism. These properties may include the differentiation stage and mutations occurring during tissue culture adaptation, the latter involving mutations that cooperate with other genetic alterations leading to the final leukemogenic alterations.

\section{E. Tumorigenicity of Factor-Independent Progenitor and Stem Cell Lines}

Several groups have shown that tumorigenicity may be a property of cells that have acquired factor-independent growth by autonomous CSF production [6-9]. Having dissected two stages of factor independence in vitro (density dependent versus autonomous growth), we investigated whether acquisition of either type of factor-independent growth was sufficient to induce tumor formation in susceptible mice or whether growth autonomy is prerequisite for tumorigenicity.

Congenic mice were injected with $10^{5}$ to $10^{8}$ cells of density-dependent or autonomous M3MuV infected FDC-P1/$\mathrm{P} 2$ progenitor cell clones. Injection of up to $10^{8}$ control cells which were either un- infected or infected with a control vector into 33 individual mice did not induce tumors within a 4-month period of observation. In contrast, all mice injected with $10^{8} \mathrm{M} 3 \mathrm{MuV}$ infected cells acquired tumors regardless of the nature of factor independence. However, the latency of tumor formation and progression in tumor weight was strikingly different from those tumors derived by nonautonomous clones as compared with autonomous clones. Four out of nine animals injected with as few as $10^{6}$ cells of the autonomous clones showed tumor formation 4 weeks after inoculation and all animals $(9 / 9)$ acquired tumors within 8 weeks time. The average tumor weight increased from $0.5 \mathrm{~g}$ at 2 weeks to more than $2.5 \mathrm{~g}$ just before death of the mice. The tumors induced by nonautonomous clones showed a significantly longer latency. Eight weeks after inoculation only $10 \%$ of injected mice (3/30) displayed tumors with an average tumor weight of $0.1 \mathrm{~g}$ and even after 12 weeks only $63 \%$ $(19 / 30)$ of the animals showed tumors. To investigate whether the pronounced longer onset of tumor formation from nonautonomous clones was due to in vivo selection for autonomous variants, we either reestablished cell lines from tumors or plated cell suspensions from tumors directly in our in vitro assay in the absence of added growth factor. As expected, all cell lines established from tumors initiated by autonomous clones showed density-independent clonability when plated in the absence of WEHI 
$\mathrm{CM}$. The cells reestablished from tumors of density-dependent clones at the time point of inoculation gave rise to either autonomous or density-dependent subclones. Interestingly, reinjection of the in vivo passaged, density-dependent subclones resulted in accelerated tumor formation. Autonomous cell growth thus is only one parameter that may lead to rapid tumorigenicity; other and as yet unknown parameters unrelated to density-independent growth are also definitely involved in accelerating tumorigenicity in vivo.

In accordance with this hypothesis, neither density-independent nor autonomous clones of the M3MuV-infected FDC-Pmix stem cell clones (15S) induced tumor formation when injected into congenic animals. Multipotent stem cells thus require further alterations for acquisition of accelerated tumorigenesis.

\section{F. Summary and Conclusions}

Tumorigenesis of hemopoietic cells and acquisition of factor independence as a consequence of aberrant growth factor release are closely correlated [5-9]. In previous work we were able to dissect two stages leading to growth factor autonomy of cells: the first step requires the secretion of the constitutively expressed CSF gene product and extracellular interaction with its cognate receptor. This requirement for external stimulation is abrogated by a second step. We were interested in characterizing the parameters that influence the conversion from nonautonomous to autonomous growth properties of hematopoietic precursor cells. The frequency with which this alteration occurs varies and correlates with the level of growth factor production. However, a significant increase of CSF production accompanying the progression to autonomy could not be detected. We thus conclude that there is no direct link between level of CSF production and acquisition of true autonomy but an indirect influence enhancing the frequen- cy of genetic alteration(s) that lead to growth autonomy.

Lang et al. [6] have suggested that the acquisition of autonomous growth occurs due to internal receptor-ligand interaction. Indeed, Keating and Williams [11] have claimed that PDGF may react with an intracellular PDGF receptor resulting in autocrine stimulation.

We therefore determined the importance of localization of the CSF molecules utilizing a pair of constructs that either result in expression of a secreted gene product (sMuV) or a mostly membrane-bound form (lMuv). In accordance with our expectation, we found a close to linear clonability of cells following infection with the construct conferring the membrane-bound form of the molecule, whereas infection with the secreted product vector showed densitydependent clonability under growth-factor selection. These data suggest that receptor-ligand interaction also may occur intracellularly, e.g., in the membranes of the endoplasmatic reticulum (ER) or at the inner face of the outer cell membrane.

Work by Browder et al. [12] is in accordance with this hypothesis. Infection of multi-CSF-dependent 32D cells with a construct encoding a modified multiCSF protein that does not direct the CSF molecule into the ER for processing did not lead to factor independence, although the gene product localized in the cytosol proved to be biologically active.

In vivo studies performed to investigate the link between acquisition of factor independence and tumorigenicity of infected progenitor cells revealed that infected cell lines of autonomous as well as nonautonomous factor independence were tumorigenic. However, second mutations also accelerated the onset of tumor formation and progression.

The most intriguing result obtained from our experiments is the striking difference in initiation of leukemogenesis in haemopoietic stem cell lines as compared with the fast progression in precursor cells. Only one out of nine infected stem cell lines tested shifted from nonauton- 
omous factor independence to true autonomy over a time period of 6 months. Inoculation of congenic mice did not induce tumor formation within the observation period when similarly infected FDC-P progenitor cells already elicited tumor formation. The stem cell lines thus appear to be more stable and less susceptible to genetic mutations than the more differentiated progenitor cell lines. Whether this is a characteristic of the undifferentiated stage or their close to normal karyotype remains to be clarified.

\section{G. Outlook}

Although it has long been clear that leukemogenesis is a multistep process, the genetic changes that are involved remain unclear. Analysis of leukemic cells from both AML and CML patients have provided evidence in support of the hypothesis that aberrant expression of growth factor genes (e.g., GM-CSF and G-CSF) may contribute to the uncontrolled growth of the leukemic cells [13, 14]. Indeed, two experimental studies have demonstrated the causal correlation of aberrant factor production with leukemogenic growth: unregulated expression of an IL-6 gene conjugated to the human Ig heavy chain gene enhancer $(\mathrm{E} \mu)$ in transgenic mice triggers generation of plasmacytoma/myeloma (Suematsu and Kishimoto, cited in [15]) and expression of IL-3 in a retroviral construct introduced in vivo via murine hematopoietic stem cells leads to a hematopoietic disorder similar to CML [12].

Although initial reports suggested that aberrant factor expression leads directly to tumorigenic growth $[6,8,9]$, the studies presented here and elsewhere $[5,7,17]$ have clearly demonstrated that secondary events are necessary for true autonomous growth and increased tumorigenic potential. These secondary events may be dependent on the autogenous factor production or may have obtained selective advantage in cells producing their own factor. The first step in the unmasking of these events has been the development of an in vitro model system, as has been described here. We hope that their ultimate genetic identification can be facilitated by insertional mutagenesis followed by selection for autonomous growth.

Acknowledgement. This work was supported by grants from the Deutsche Forschungsgemeinschaft (DFG Os 31-12) and the Deutsche Krebshilfe.

\section{References}

1. Metcalf D (1984) The haemopoietic colony stimulating factors. Elsevier, Amsterdam

2. Spooncer E, Heyworth CM, Dunn A, Dexter TM (1986) Self-renewal and differentiation of interleukin-3-dependent multipotent stem cells are modulated by stromal cells and serum factors. Differentiation 31:111-118

3. Dexter TM, Garland J, Scott D, Scolnick E, Metcalf D (1980) Growth of factor-dependent hemopoietic precursor cell lines. $J$ Exp Med 152:1036-1047

4. Stocking C, Kollek R, Bergholz U, Ostertag W (1985) Long terminal repeat sequences impart hematopoietic transformation properties to the myeloproliferative sarcoma virus. Proc Natl Acad Sci USA 79: $5746-5750$

5. Laker C, Stocking C, Bergholz U, Hess N, DeLamarter JF, Ostertag W (1987) Autocrine stimulation after transfer of the granulocyte-macrophage colony stimulating factor gene and autonomous growth are distinct but interdependent steps in the oncogenic pathway. Proc Natl Acad Sci USA $84: 8458-8462$

6. Lang RA, Metcalf D, Gough NM, Dunn AR, Gonda TJ (1985) Expression of a hemopoietic growth factor cDNA in a factor-dependent cell line results in autonomous growth and tumorigenicity. Cell 43:531-542

7. Wong PMC, Chung S-W, Nienhuis A (1987) Retroviral transfer and expression of the interleukin-3 gene in hemopoietic cells. Genes Dev 1:358-365

8. Yamada G, Kitamura Y, Sonoda $H$, Harada H, Taki S, Mulligan RC, Osawa $\mathrm{H}$, Diamantstein $\mathrm{T}$, Yokoyama $\mathrm{S}$, Taniguchi T (1987) Retroviral expression 
of the human IL-2 gene in a murine $T$ cell line results in cell growth autonomy and tumorigenicity. EMBO J 6:2705-2709

9. Hapel AJ, Vande Woude G, Campbell HD, Young IG, Robins T (1986) Generation of an autocrine leukaemia using a retroviral expression vector carrying the interleukin-3 gene. Lymphokine Res 5: 249-254

10. Haeuptle MT, Feint N, Gough NM, Dobberstein B (1989) A tripartite structure of the signals that determine protein insertion into the ER membrane. J Cell Biol 108: $1227-1236$

11. Keating MT, Williams LT (1988) Autocrine stimulation of intracellular PDGF receptors in v-sis-transformed cells. Science 239:914-916

12. Browder TM, Abrams JS, Wong PMC, Nienhuis AW (1989) The mechanism of autocrine stimulation in hematopoietic cells producing interleukin 3 after retroviral mediated transfer. Mol Cell Biol 9:204-213

13. Young DC, Griffin JD (1986) Autocrine secretion of GM-CSF in acute myeloblastic leukemia. Blood 68:1178-1181

14. Klein H, Becker R, Lindemann A, Oster W, Schleiermacher E, Souza L, Mertels- mann R, Herrmann F (1989) Synthesis of granulocyte colony-stimulating factor and its requirement for terminal divisions in chronic myelogenous leukaemia. J Exp Med (in press)

15. Hirano T, Kishimoto (1989) Interleukin6. In: Sporn MB, Roberts AB (eds) Peptide growth factors and their receptors. Springer, Berlin Heidelberg New York, chap 14 (Handbook of Experimental Pharmacology vol 95, part 1)

16. Spooncer E, Katsuno M, Hampson I, Dexter TM, Just U, Stocking C, Kluge N, Ostertag W (1989) Biological effects of retroviral transfection of the murine interleukin-3 gene into FDC-P mix cells. In: Shen-ong GLC, Potter M, Copeland NG (eds) Mechanisms in myeloid tumor regenesis. Springer, Berlin Heidelberg New York (Current topics in microbiology and immunology, vol 149)

17. Johnson A, Betsholtz C, Heldin C-H, Westermark B (1986) The phenotypic characteristics of simian sarcoma virustranformed human fibroblasts suggest that the v-sis gene product acts solely as a PDGF receptor agonist in cell transformation. EMBO J 5:1535-1541 